\title{
Genetic characterization of the 28 maize landraces in Paraná State
}

\section{Caracterização genética de 28 populações de milho crioulo no Estado do Paraná}

\author{
Sara Regina Silvestrin Rovaris ${ }^{1 *}$; Pedro Mário de Araújo ${ }^{2}$; \\ Deoclécio Domingos Garbuglio ${ }^{2}$; Cleber Vinicius Guiaretta de Azevedo ${ }^{3}$; \\ Cássio Egídio Cavenaghi Prete ${ }^{4}$
}

\begin{abstract}
The characterization of maize landraces is extremely important in breeding programs for use of these genotypes as sources of genetic variability. The objective of this study was to quantitatively characterize 28 populations of maize landraces from the state of Paraná using the estimates of the effects of varieties and heterosis parents and the general combining ability, thereby assessing the main agronomic traits. In the crop of 2008/09, 56 inter-varietal hybrids, obtained through a topcross, 28 populations of maize landraces and three check varieties were evaluated for female flowering (FF), plant height (PH), ear height $(\mathrm{EH})$ and grain mass (GY). The treatments were evaluated in a randomized block design, with two replications, at three Paraná State locations: the Experimental Center of the Agronomic Institute of Paraná in Londrina (IAPAR) and the experimental units of Pato Branco and Ponta Grossa. The data were submitted to an analysis of variance, considering a fixed model for genotypes and a random model for environments; the averages grouped by the Scott-Knott test, along with intersections of topcrosses, were analyzed according to a readapted model proposed by Oliveira et al. (1997). According to estimates of the parental effects, the GI 133 population showed the most promising estimates for all characteristics. The GI 088 and GI 173 populations stood out with promising estimates of the effects of heterosis. The conclusion is that the populations GI 133 and GI 173 may be indicated for recurrent selection programs or participation in obtaining composites.
\end{abstract}

Key words: Heterosis effects. Variety effects. Pre-breeding. Zea mays L.

\section{Resumo}

A caracterização de milho crioulo ou variedades locais de milho é de suma importância em programas de melhoramento, para utilização desses genótipos como fonte de variabilidade genética. O objetivo deste trabalho foi realizar a caracterização quantitativa de 28 populações de milho crioulo, oriundas do estado do Paraná, pelas estimativas dos efeitos de variedades e heterose de parentais e capacidade geral de combinação, avaliando as principais características agronômicas. Na safra de 2008/09, 56 híbridos intervarietais obtidos por meio de um cruzamento "topcross", 28 populações de milho crioulo e mais três testemunhas, foram avaliados quanto ao florescimento feminino (FF), altura de planta (PH) e de espiga (EH) e massa de grãos (GY). Os tratamentos foram avaliados em delineamento de blocos ao acaso, com duas repetições, em três locais do estado de Paraná: no Centro Experimental do Instituto Agronômico do

${ }^{1} \mathrm{Dr}^{\mathrm{a}}$ em Agricultura Tropical e Subtropical Genética, Melhoramento Vegetal e Biotecnologia, Instituto Agronômico, IAC, Campinas, SP, Brasil. E-mail: sara_rsr@yahoo.com.br

2 Pesquisadores, Instituto Agronômico do Paraná, IAPAR, Londrina, PR, Brasil. E-mail: pmaraujo@iapar.br; ddgarbuglio@iapar.br

3 Discente de Doutorado, Programa de Pós-Graduação, Faculdade de Ciências Agrárias e Veterinárias de Jaboticabal, UNESP, Jaboticabal, SP, Brasil. E-mail: cleber_az@yahoo.com.br

4 Prof. Dr., Universidade Estadual de Londrina, UEL, Centro de Ciências Agrárias, Departamento de Agronomia, Londrina, PR, Brasil. E-mail: cassio@uel.br

* Author for correspondence 
Paraná em Londrina (IAPAR) e nas unidades experimentais de Pato Branco e Ponta Grossa. Os dados foram submetidos às análises de variância, considerando-se o modelo fixo para genótipos e aleatório para ambientes, sendo as médias agrupadas pelo Teste de Scott-Knott e os cruzamentos "topcrosses" foram analisados conforme o modelo readaptado proposto por Oliveira et al. (1997). Pelas estimativas dos efeitos de variedades, a população GI 133 apresentou as estimativas mais promissoras para todas as características. As populações GI 088 e GI 173 destacaram-se pelas estimativas promissoras dos efeitos de heterose. Conclui-se que as populações GI 133 e GI 173 podem ser indicadas para programas de seleção recorrente ou participação para formação de compostos.

Palavras-chave: Efeitos de variedade. Efeitos de heterose. Pré-melhoramento. Zea mays L.

\section{Introduction}

Maize landraces, or maize local varieties, were originated through the direct action of successive generations of family farmers and indigenous communities by crossing old and even recent genotypes, or simply through intrapopulation selection of plants adapted to their farming systems (FERREIRA et al., 2009). Although maize landraces are less productive than commercial cultivars, they have important specific characteristics that justify their evaluation for pre-breeding programs, such as the high potential for adaptation and cultivation in rural environments that have water deficiencies, nutrient shortages, and excess acidity or alkalinity in the soil. These populations are considered a source of genetic variability that can be exploited in the search for genes that are tolerant and/or resistant to biotic and abiotic factors (ARAÚJO; NASS, 2002).

The characterization of maize landraces access to germplasm banks through an evaluation of these variables encourages their use and contributes to the identification of divergent populations with genetic variability, which may be useful for the preliminary prediction of crosses that optimize heterosis (MIRANDA et al., 2003).

Topcrosses are generally used when there is no information available about the genotypes under study. These crosses also allow for the selection of populations for further research of recurrent intra- or inter-population selection (reciprocal recurrent selection), forming compounds or the commercial use of $F_{1}$ (GARBUGLIO; ARAÚJO, 2006; HALLAUER; MIRANDA FILHO, 1988). The topcrosses are easy to achieve, thus allowing the evaluation of a large number of genotypes and obtaining important parameters and genetic effects during selection (CRUZ et al., 2004).

Theoretical and experimental evaluations of topcrosses have been presented with the objective of obtaining information about the frequency of favorable alleles, their genetic basis, their general or specific combining ability, the yield "per se," the number of testers and the genealogy between the evaluated genotypes (SEIFERT et al., 2006; FERREIRA et al., 2009; CARPENTIERI-PÍPOLO et al., 2010, CANCELLIER et al., 2011; CLOVIS et al., 2015). Furthermore, topcrosses are widely used in maize breeding programs, with the objective of studying the most diverse agronomic characteristics. Cancellier et al. (2011) evaluated the potential for 70 topcross hybrids selected for plant height, ear height, ear length and ear diameter, 100 seed weight, hectoliter weight and grain yield, observing that nineteen lines presented a set of characteristics that are favorable to the development of commercial genotypes in the production of grain in the southern Tocantins.

Ferreira et al. (2009) reported that few studies have been carried out to evaluate the potential of these maize landrace varieties in crosses. The same authors performed a topcrosses cross between 31 varieties of maize landraces with two hybrids and obtained high estimates of combining ability, allowing for the indication of these genotypes in the synthesis of compounds. Kostetzer et al. (2009) evaluated maize varieties using a partial diallel (11 x 5) and verified that the MC 45 and IAPAR 50 varieties presented promising genetic parameter 
estimates for all variables. The present work aimed to characterize 28 maize landrace populations for their estimates of variety and heterosis effects and their general combining ability in terms of their main agronomic characteristics.

\section{Materials and Methods}

In the growing season of 2007/2008, 56 topcross hybrids were obtained by a partial diallel $(28 \times 2)$ between 28 maize landrace populations and two maize tester populations (Table 1).

Table 1. Description of the 28 maize landrace populations, 2 tester populations and check varieties according to the origin, characteristics of the grain, days for female flowering (FF) and one-thousand grain weight (P 1000G).

\begin{tabular}{|c|c|c|c|c|c|}
\hline \multicolumn{6}{|c|}{ Maize Landrace Populations } \\
\hline \multirow{2}{*}{ Genotypes } & \multirow{2}{*}{ Origin } & \multicolumn{2}{|c|}{ Characteristics of the grain } & \multirow{2}{*}{$\begin{array}{c}\text { FF } \\
\text { (days) } \\
\end{array}$} & \multirow{2}{*}{$\begin{array}{c}\text { P 1000G } \\
(\mathrm{g})\end{array}$} \\
\hline & & Type & Color & & \\
\hline GI 002 & Amarilo Opaco Amiláceo & flint & yellow & 76 & 161.38 \\
\hline GI 006 & Asteca Lupionópolis & dentate & orange & 72 & 293.44 \\
\hline GI 008 & Asteca Nova Esperança & dentate & yellow & 72 & 314.24 \\
\hline GI 018 & Cravo n 1 & dentate & orange & 68 & 263.44 \\
\hline GI 028 & Mato Grosso São João & dentate & orange & 68 & 341.18 \\
\hline GI 036 & Roxo asteca (G 5) & dentate & red & 70 & 329.04 \\
\hline GI 045 & Tupi Pytá Sopé & dentate & orange & 68 & 298.02 \\
\hline GI 048 & Composto Indonésia & flint & orange & 64 & 223.08 \\
\hline GI 049 & Palmeira (1) & dentate & yellow & 64 & 287.10 \\
\hline GI 088 & RGS III & dentate & yellow & 64 & 314.40 \\
\hline GI 101 & Cristal Paraguai & flint & white & 75 & 210.94 \\
\hline GI 104 & Peróla & semi- flint & white & 68 & 249.16 \\
\hline GI 105 & Tupi Moroti & dentate & white & 76 & 190.12 \\
\hline GI 133 & CIMMYT & dentate & orange & 62 & 274.94 \\
\hline GI 135 & Milho dos índios & dentate & red & 75 & 322.64 \\
\hline GI 140 & Astequinha & dentate & yellow & 68 & 318.16 \\
\hline GI 148 & General & dentate & orange & 67 & 289.42 \\
\hline GI 149 & Linha Paraná & dentate & yellow & 69 & 337.46 \\
\hline GI 150 & Linha Paraná & dentate & white & 68 & 256.92 \\
\hline GI 151 & Asteca Baixo & dentate & yellow & 70 & 326.88 \\
\hline GI 152 & Amarelão & dentate & yellow & 68 & 254.42 \\
\hline GI 153 & Macaco & dentate & yellow & 68 & 363.52 \\
\hline GI 154 & Tabuinha & dentate & yellow & 70 & 303.76 \\
\hline GI 155 & Dente de Rato & dentate & yellow & 70 & 279.76 \\
\hline GI 156 & Caiano Sobralia & dentate & yellow & 68 & 321.92 \\
\hline GI 157 & Asteca Branco & dentate & white & 75 & 336.74 \\
\hline GI 158 & Antigo Maia & dentate & yellow & 69 & 340.26 \\
\hline GI 173 & Palotina - PR & dentate & yellow & 68 & 377.68 \\
\hline \multicolumn{6}{|c|}{ Check Varieties } \\
\hline IPV 2122 & IAPAR & dentate & orange & 65 & 312.54 \\
\hline IPR 114 & IAPAR & dentate & orange & 71 & 331.81 \\
\hline IPR 115 & IAPAR & dentate & orange & 65 & 309.52 \\
\hline \multicolumn{6}{|c|}{ Testers } \\
\hline PC 0201 & IAPAR & dentate & yellow & 65 & 319.44 \\
\hline PC 0202 & IAPAR & dentate & orange & 65 & 321.62 \\
\hline
\end{tabular}

GI: germplasm introduced. 
The performance of the 56 hybrids, the tester populations, the maize landrace populations per se and the three check varieties, IPV 2122, IPR 114 and IPR 115, were evaluated during the 2008/2009 harvest at three locations in the state of Paraná: Londrina, Pato Branco and Ponta Grossa. The following agronomic traits were evaluated: female flowering (FF) in days, from emergency to stigma style emission in $50 \%$ of the plants of the plot; plant height $(\mathrm{PH})$ in centimeters, measured from the soil surface to the curvature of the flag leaf; ear height $(\mathrm{EH})$ in centimeters, measured from the soil surface to the point of insertion of the upper ear; and grain yield (GY) resulting from the mechanical threshing of the plot ears, measured with an electronic scale in $\mathrm{kg} \mathrm{ha}^{-1}$ (corrected for 14\% humidity and ideal stand). For GY, only the data from Pato Branco and Ponta Grossa were considered, as those of Londrina were discarded due to the occurrence of drought.

The experimental design was a randomized complete block design, with two replications in three environments. The plot consisted two $5 \mathrm{~m}$ long rows, spaced $0.80 \mathrm{~m}$ apart between rows and 0.2 $\mathrm{m}$ between plants, totaling 25 plants per row after thinning.

At all locations, fertilization was performed using $350 \mathrm{~kg} \mathrm{ha}^{-1}$ of NPK (8:28:16), and the topdressing fertilization was performed using $60 \mathrm{~kg} \mathrm{ha}^{-1}$ of $\mathrm{N}$ supplied as urea.

The data were submitted to individual and joint analyses of variance, according to the simple factorial model with additional check varieties, with fixed genotypes and as random environments (CRUZ et al., 2004). The means were compared using the Scott-Knott test (1974) at 5\% probability.

The topcross hybrids were evaluated according to the model readaptation of Miranda Filho and Geraldi (1984), proposed by Oliveira et al. (1987), applicable to partial diallels, considering several environments. The model for diallel crosses includes two population groups and the hybrids resulting from inter-varietal crosses of distinct groups. When the assays were repeated in several environments, the model was readjusted by Oliveira et al. (1987) as follows:

$$
\begin{gathered}
Y_{\mathrm{ij}}=\bar{\mu}+\alpha d+\ell_{\mathrm{i}} 1 / 2\left(\mathrm{v}_{\mathrm{i}}+\mathrm{v}_{\mathrm{j}^{\prime}}\right)+\theta\left(\overline{\mathrm{h}}+\mathrm{h}_{\mathrm{i}}+\mathrm{h}_{\mathrm{j}}+\mathrm{s}_{\mathrm{ji}}\right)^{\prime}+ \\
+\alpha \ell \mathrm{d}_{\mathrm{i}}+1 / 2\left(\ell \mathrm{v}_{\mathrm{ij}}+\ell \mathrm{v}_{\mathrm{ij}}\right)+\theta\left(\ell \overline{\mathrm{h}}+\ell \mathrm{h}_{\mathrm{i}}+\ell \mathrm{h}_{\mathrm{j}}+\ell \mathrm{s}_{\mathrm{jj}}\right) \bar{\varepsilon}_{\mathrm{ij} j^{\prime}},
\end{gathered}
$$

where $\mathrm{Y}_{\mathrm{ij}}$ : = the average of hybrids resulting from crosses between the jth group 1 population and the $\mathrm{J}$ population of group 2 in the i-th environment; $\bar{\mu}=$ average of the averages of both groups for all environments; $\ell_{\mathrm{i}}=$ the effect of the $\mathrm{i}$-th environment; $\alpha=$ of value 0 , and -1 and 1 for hybrids for the group of higher average genotypes and the lower average group, respectively; $\mathrm{d}=$ the difference between the two groups; $v_{i}$ and $v_{j}=$ the effect of the variety of groups (1) and (2), respectively; $\theta=$ the value 0 for variety and 1 for hybrids; $\bar{h}=$ effect of the mean heterosis of crosses; $h_{i}$ and $h_{j}=$ the effect of the heterosis of the variety of groups (1) and (2), respectively; $\mathrm{s}_{\mathrm{ij}}$ : = the effect of the specific heterosis of the jj"-th hybrid; and $\bar{\varepsilon}_{\mathrm{ijj}}=$ experimental error associated with hybrids or parental traits.

Statistical analyses were performed using the packages GENES (CRUZ, 2006) and MAPGEN (FERREIRA, 1993).

The estimate of the general combining ability $\left(\hat{\mathrm{g}}_{\mathrm{i}}\right)$ was calculated using the following expression (GRIFFING, 1956):

$$
\hat{g}_{i}=\frac{v_{i}}{2}+h_{i}
$$

where $\mathrm{v}_{\mathrm{i}}=$ variety effect and $\mathrm{h}_{\mathrm{i}}=$ heterosis effect.

\section{Results and Discussion}

The joint analyses of variance detected significant differences $(p<0.05$ and $p<0.01)$ for FF, PH and GY for treatments (T) and genotypes 
(G), inferring the differences in performance among the evaluated genotypes (Table 2). The effects of $\mathrm{T}$ $x$ E were significant $(\mathrm{p}<0.05)$ only for $\mathrm{PH}, \mathrm{EH}$ and GY, so their means are presented by location. When there is significance in this interaction, this indicates that there is a possibility of a genotype presenting excellent performance in one environment, while not being influenced by the recommendation of cultivars for wide adaptability in another (CLOVIS et al., 2015).

The coefficients of variation obtained in this study ranged from low to medium, indicating that the experimental design was satisfactory in controlling environmental variation, while allowing reliable data to be obtained (FRITSCHE-NETO et al., 2012; PIMENTEL-GOMES, 2000).

Table 2. Mean squares (MS) in the joining analysis for female flowering (FF), plant height (PH), ear height (EH) and grain yield (GY) of topcross hybrids and maize landrace populations. Londrina, Pato Branco and Ponta Grossa - PR. Season 2008/09.

\begin{tabular}{|c|c|c|c|c|c|c|}
\hline \multirow{3}{*}{ Source of variation } & \multicolumn{5}{|c|}{ Mean Squares (MS) } & \multirow{3}{*}{$\begin{array}{c}\text { MS } \\
\text { GY } \\
\left(\mathrm{kg} \mathrm{ha}^{-1}\right)\end{array}$} \\
\hline & df & $\mathrm{FF}$ & $\mathrm{PH}$ & $\mathrm{EH}$ & \multirow{2}{*}{ df' } & \\
\hline & \multicolumn{2}{|c|}{ (days) } & \multicolumn{2}{|c|}{$(\mathrm{cm})$} & & \\
\hline Treatments (T) & 88 & $42.26 * *$ & $1025.61^{*}$ & $471.41^{\mathrm{ns}}$ & 88 & $11531242.21 * *$ \\
\hline Genotypes (G) & 85 & $39.41 * *$ & $1056.22 *$ & $480.03^{\mathrm{ns}}$ & 85 & $11396954.9 * *$ \\
\hline Check Varieties (C) & 2 & $10.88^{\mathrm{ns}}$ & $236.16^{\mathrm{ns}}$ & $96.05^{\mathrm{ns}}$ & 2 & $6970549 *$ \\
\hline Groups (Gru) & 1 & $347.57 *$ & $2.74^{\mathrm{ns}}$ & $489.48^{\mathrm{ns}}$ & 1 & $32067049.76^{*}$ \\
\hline Environments (E) & 2 & $6360.20 * *$ & $53882.72 * *$ & $75054.76^{* *}$ & 1 & $4454471.02 *$ \\
\hline Tx E & 176 & $16.03^{\mathrm{ns}}$ & $706.07 *$ & $448.28 *$ & 88 & $2677926.03 *$ \\
\hline $\mathrm{G} \times \mathrm{E}$ & 170 & $16.33^{\mathrm{ns}}$ & $725.25^{\mathrm{ns}}$ & $457.37^{\mathrm{ns}}$ & 85 & $2764161.94^{\text {ns }}$ \\
\hline $\mathrm{CxE}$ & 4 & $2.55^{\mathrm{ns}}$ & $176.58^{\mathrm{ns}}$ & $208.55^{\mathrm{ns}}$ & 2 & $167510.33^{\mathrm{ns}}$ \\
\hline Gru x E & 2 & $17.33^{\mathrm{ns}}$ & $134.18^{\mathrm{ns}}$ & $155.13^{\mathrm{ns}}$ & 1 & $368705.17^{\mathrm{ns}}$ \\
\hline Error & 264 & 15.38 & 205.53 & 132.47 & 176 & 1053914.39 \\
\hline General means & & 75 & 219 & 122 & & 5345 \\
\hline Genotype means & & 75 & 211 & 118 & & 5289 \\
\hline $\mathrm{C}$ means & & 75 & 205 & 110 & & 6952 \\
\hline $\mathrm{CV}(\%)$ & & 5.00 & 6.78 & 9.72 & & 19.20 \\
\hline
\end{tabular}

df': Degrees of freedom considering two environments.

$* *$, *: significant at $5 \%$ and $1 \%$ probability by test $\mathrm{F}$.

ns: no significant at $5 \%$ and $1 \%$ probability by test $\mathrm{F}$.

For female flowering (FF), most of the hybrids (92\%) had averages similar to the commercial ones being considered as early genotypes (Table 3). Regarding plant height $(\mathrm{PH})$ in Londrina, the test did not stratify the genotypes into groups. The averages presented in Pato Branco were divided into two groups ( $a$ and b), whereas in Ponta Grossa, the genotypes had very different $\mathrm{PH}$ means and were separated into 11 groups. For AE, three groups ( $a, b$ and c) were observed in Londrina, two groups in Pato Branco ( $a$ and $b$ ), and five groups in Ponta Grossa (a-e). For grain yield (GY), the hybrids in Pato Branco were divided into four groups, and only
19 hybrids did not differ from the means presented by the evaluated varieties. In Ponta Grossa, the hybrids were divided into three groups ( $a, b$ and $c$ ) and some hybrids, such as GI 088 x TC1, GI 133 x TC1, GI 045 x TC2, GI 148 x TC2 and GI173 x $\mathrm{TC} 2$, were shown to have similar performance to check variety IPR 115 (group a). GI 088 x TC1, GI 133 x TC1, GI 148 x TC2 and GI 173 x TC2 hybrids exhibited satisfactory performance for GY in both environments. All these results indicate that these genotypes of maize landraces exhibit a performance different from the traits in environments where testing was done and that this fact informs selection. 
Table 3. Means for female flowering (FF), plant height (PH), ear height (EH) and grain yield (GY) of topcross hybrids and of check varieties in three environments of Paraná state, Londrina (LD), Pato Branco (PB) and Ponta Grossa (PG). Season 2008/09.

\begin{tabular}{|c|c|c|c|c|c|c|c|c|c|c|c|c|c|c|c|c|c|c|}
\hline \multirow{3}{*}{ Hybrids } & \multirow{2}{*}{\multicolumn{2}{|c|}{$\begin{array}{c}\text { FF } \\
\text { (days) }\end{array}$}} & \multicolumn{6}{|c|}{$\mathrm{PH}$} & \multicolumn{6}{|c|}{$\mathrm{EH}$} & \multirow{2}{*}{\multicolumn{4}{|c|}{$\frac{\mathrm{GY}}{\left(\mathrm{kg} \mathrm{ha}^{-1}\right)}$}} \\
\hline & & & \multicolumn{6}{|c|}{$(\mathrm{cm})$} & \multicolumn{6}{|c|}{$(\mathrm{cm})$} & & & & \\
\hline & \multicolumn{2}{|c|}{ Means } & LD & & $\mathrm{PB}$ & & PC & & LD & & $\mathrm{PE}$ & & PC & & \multicolumn{2}{|c|}{ PB } & \multicolumn{2}{|l|}{ PG } \\
\hline GI $002 \times \mathrm{TC} 1$ & 76 & $\mathrm{c}$ & 208 & $\mathrm{a}$ & 193 & $\mathrm{~b}$ & 205 & $\mathrm{i}$ & 118 & $\mathrm{c}$ & 93 & $\mathrm{~b}$ & 120 & $\mathrm{~d}$ & 5652 & $\mathrm{~b}$ & 4412 & $\mathrm{c}$ \\
\hline GI $006 \times$ TC 1 & 73 & $\mathrm{c}$ & 235 & $\mathrm{a}$ & 178 & $\mathrm{~b}$ & 243 & $\mathrm{e}$ & 123 & $\mathrm{~b}$ & 90 & $\mathrm{~b}$ & 158 & $\mathrm{~b}$ & 6091 & $b$ & 6903 & b \\
\hline GI $008 \times$ TC1 & 76 & $\mathrm{c}$ & 240 & $\mathrm{a}$ & 200 & $\mathrm{a}$ & 251 & $\mathrm{~d}$ & 140 & $\mathrm{a}$ & 103 & $\mathrm{~b}$ & 140 & $\mathrm{c}$ & 6756 & $\mathrm{a}$ & 6401 & b \\
\hline GI $018 \times$ TC 1 & 75 & $\mathrm{c}$ & 253 & $\mathrm{a}$ & 205 & $\mathrm{a}$ & 245 & $\mathrm{e}$ & 160 & $\mathrm{a}$ & 125 & $\mathrm{a}$ & 136 & $\mathrm{c}$ & 6109 & $\mathrm{~b}$ & 9178 & $\mathrm{a}$ \\
\hline GI $028 \times$ TC1 & 76 & $\mathrm{c}$ & 233 & $\mathrm{a}$ & 180 & $\mathrm{~b}$ & 226 & $\mathrm{~g}$ & 143 & $\mathrm{a}$ & 90 & $\mathrm{~b}$ & 123 & $\mathrm{~d}$ & 5779 & $\mathrm{~b}$ & 6954 & b \\
\hline GI $036 \times$ TC1 & 76 & $\mathrm{c}$ & 235 & $\mathrm{a}$ & 208 & $\mathrm{a}$ & 250 & d & 135 & $\mathrm{a}$ & 100 & $\mathrm{~b}$ & 153 & b & 7019 & $\mathrm{a}$ & 7465 & b \\
\hline GI $045 \times$ TC1 & 74 & $\mathrm{c}$ & 233 & $\mathrm{a}$ & 180 & $\mathrm{~b}$ & 206 & $\mathrm{i}$ & 138 & $\mathrm{a}$ & 90 & $\mathrm{~b}$ & 108 & $\mathrm{e}$ & 5991 & $\mathrm{~b}$ & 6329 & b \\
\hline GI $048 \times$ TC1 & 75 & $\mathrm{c}$ & 220 & $\mathrm{a}$ & 195 & $\mathrm{~b}$ & 231 & $\mathrm{f}$ & 128 & b & 110 & $\mathrm{a}$ & 138 & c & 5079 & c & 9329 & $\mathrm{a}$ \\
\hline GI 049 x TC1 & 75 & $\mathrm{c}$ & 250 & $\mathrm{a}$ & 203 & $\mathrm{a}$ & 233 & $\mathrm{f}$ & 138 & $\mathrm{a}$ & 105 & $\mathrm{a}$ & 140 & $\mathrm{c}$ & 5064 & $\mathrm{c}$ & 7419 & b \\
\hline GI $088 \times$ TC1 & 75 & $\mathrm{c}$ & 130 & $\mathrm{a}$ & 195 & $\mathrm{~b}$ & 238 & e & 135 & a & 103 & $\mathrm{~b}$ & 123 & c & 6589 & a & 8289 & $\mathrm{a}$ \\
\hline GI $101 \times$ TC1 & 77 & $\mathrm{c}$ & 215 & $\mathrm{a}$ & 208 & $\mathrm{a}$ & 234 & $\mathrm{f}$ & 133 & $\mathrm{~b}$ & 133 & $\mathrm{a}$ & 130 & $d$ & 5886 & $\mathrm{~b}$ & 5089 & $\mathrm{c}$ \\
\hline GI $104 \times$ TC1 & 76 & $\mathrm{c}$ & 243 & $\mathrm{a}$ & 215 & $\mathrm{a}$ & 242 & e & 145 & $\mathrm{a}$ & 118 & $\mathrm{a}$ & 139 & $\mathrm{c}$ & 5323 & $\mathrm{~b}$ & 6103 & b \\
\hline GI $105 \times$ TC1 & 81 & $\mathrm{~b}$ & 223 & $\mathrm{a}$ & 180 & $\mathrm{~b}$ & 239 & $\mathrm{e}$ & 143 & $\mathrm{a}$ & 93 & $\mathrm{~b}$ & 146 & & 5630 & $\mathrm{~b}$ & 8633 & $\mathrm{a}$ \\
\hline GI $133 \times$ TC1 & 73 & $\mathrm{c}$ & 220 & a & 185 & $\mathrm{~b}$ & 215 & $h$ & 108 & $\mathrm{c}$ & 98 & $\mathrm{~b}$ & 113 & & 6522 & $\mathrm{a}$ & 7860 & $\mathrm{a}$ \\
\hline GI $135 \mathrm{x}$ TC1 & 77 & $\mathrm{c}$ & 245 & $\mathrm{a}$ & 208 & $\mathrm{a}$ & 267 & $\mathrm{~b}$ & 143 & $\mathrm{a}$ & 118 & $\mathrm{a}$ & 161 & b & 6280 & $\mathrm{~b}$ & 5150 & $\mathrm{c}$ \\
\hline GI $140 \times \mathrm{TC} 1$ & 76 & $\mathrm{c}$ & 215 & $\mathrm{a}$ & 225 & $\mathrm{a}$ & 241 & $\mathrm{e}$ & 133 & b & 115 & $\mathrm{a}$ & 140 & c & 6187 & $\mathrm{~b}$ & 6672 & b \\
\hline GI $148 \times$ TC1 & 74 & $\mathrm{c}$ & 233 & $\mathrm{a}$ & 218 & $\mathrm{a}$ & 239 & e & 133 & $\mathrm{~b}$ & 120 & $\mathrm{a}$ & 131 & $\mathrm{~d}$ & 6824 & $\mathrm{a}$ & 9271 & $\mathrm{a}$ \\
\hline GI $149 \times \mathrm{TC} 1$ & 75 & $\mathrm{c}$ & 245 & $\mathrm{a}$ & 233 & $\mathrm{a}$ & 226 & $\mathrm{~g}$ & 145 & $\mathrm{a}$ & 130 & $\mathrm{a}$ & 125 & $d$ & 7104 & $\mathrm{a}$ & 7061 & b \\
\hline GI $150 \times$ TC1 & 79 & $\mathrm{~b}$ & 243 & $\mathrm{a}$ & 208 & $\mathrm{a}$ & 210 & $\mathrm{i}$ & 130 & $b$ & 108 & $\mathrm{a}$ & 126 & d & 5774 & b & 7067 & $\mathrm{~b}$ \\
\hline GI $151 \times \mathrm{TC} 1$ & 73 & $\mathrm{c}$ & 235 & $\mathrm{a}$ & 188 & $\mathrm{~b}$ & 246 & $\mathrm{e}$ & 130 & b & 98 & $\mathrm{~b}$ & 143 & & 3670 & d & 6503 & b \\
\hline GI 152 x TC1 & 73 & $\mathrm{c}$ & 248 & $\mathrm{a}$ & 198 & $\mathrm{~b}$ & 244 & e & 143 & $\mathrm{a}$ & 85 & $\mathrm{~b}$ & 126 & d & 7040 & $\mathrm{a}$ & 7441 & b \\
\hline GI $153 \times$ TC 1 & 75 & $\mathrm{c}$ & 238 & $\mathrm{a}$ & 200 & $\mathrm{a}$ & 240 & $\mathrm{e}$ & 138 & a & 110 & $\mathrm{a}$ & 130 & $d$ & 5710 & b & 9283 & $\mathrm{a}$ \\
\hline GI $154 \times$ TC1 & 79 & $\mathrm{~b}$ & 245 & $\mathrm{a}$ & 208 & $\mathrm{a}$ & 247 & e & 148 & $\mathrm{a}$ & 113 & $\mathrm{a}$ & 126 & d & 6832 & $\mathrm{a}$ & 6532 & b \\
\hline GI $155 \times$ TC1 & 78 & $\mathrm{c}$ & 223 & $\mathrm{a}$ & 213 & $\mathrm{a}$ & 233 & $\mathrm{f}$ & 130 & $\mathrm{~b}$ & 120 & $\mathrm{a}$ & 121 & d & 6390 & b & 6981 & b \\
\hline GI $156 \times$ TC1 & 74 & $\mathrm{c}$ & 225 & $\mathrm{a}$ & 208 & $\mathrm{a}$ & 219 & $\mathrm{~h}$ & 130 & $\mathrm{~b}$ & 98 & $\mathrm{~b}$ & 121 & 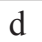 & 6647 & $\mathrm{a}$ & 6620 & b \\
\hline GI $157 \times$ TC1 & 79 & $\mathrm{~b}$ & 238 & $\mathrm{a}$ & 183 & $\mathrm{~b}$ & 217 & $\mathrm{~h}$ & 140 & $\mathrm{a}$ & 93 & $\mathrm{~b}$ & 118 & d & 6014 & $\mathrm{~b}$ & 4563 & c \\
\hline GI $158 \times$ TC1 & 76 & $\mathrm{c}$ & 233 & $\mathrm{a}$ & 193 & $\mathrm{~b}$ & 219 & $\mathrm{~h}$ & 130 & $\mathrm{~b}$ & 85 & $\mathrm{~b}$ & 119 & $d$ & 6742 & a & 7378 & b \\
\hline GI $173 \times$ TC1 & 74 & $\mathrm{c}$ & 243 & $\mathrm{a}$ & 190 & $\mathrm{~b}$ & 215 & $\mathrm{~h}$ & 140 & $\mathrm{a}$ & 100 & $\mathrm{~b}$ & 164 & b & 5443 & $\mathrm{~b}$ & 6775 & b \\
\hline GI $002 \times$ TC 2 & 76 & $\mathrm{c}$ & 243 & $\mathrm{a}$ & 180 & $\mathrm{~b}$ & 219 & $\mathrm{~h}$ & 158 & $\mathrm{a}$ & 85 & $\mathrm{~b}$ & 138 & $\mathrm{c}$ & 3008 & d & 6452 & b \\
\hline GI 006 x TC2 & 78 & $\mathrm{c}$ & 235 & $\mathrm{a}$ & 200 & $\mathrm{a}$ & 221 & $\mathrm{~h}$ & 140 & $\mathrm{a}$ & 100 & $\mathrm{~b}$ & 121 & d & 7546 & $\mathrm{a}$ & 4502 & c \\
\hline GI $008 \times$ TC2 & 76 & $\mathrm{c}$ & 258 & $\mathrm{a}$ & 198 & $\mathrm{~b}$ & 240 & $\mathrm{e}$ & 163 & $\mathrm{a}$ & 98 & $\mathrm{~b}$ & 133 & $\mathrm{~d}$ & 6661 & $\mathrm{a}$ & 5961 & b \\
\hline GI $018 \times$ TC2 & 73 & $\mathrm{c}$ & 253 & $\mathrm{a}$ & 188 & b & 219 & $\mathrm{~h}$ & 160 & $\mathrm{a}$ & 88 & $\mathrm{~b}$ & 115 & e & 7623 & $\mathrm{a}$ & 6316 & b \\
\hline GI $028 \times$ TC2 & 74 & $\mathrm{c}$ & 233 & $\mathrm{a}$ & 173 & $\mathrm{~b}$ & 236 & $\mathrm{f}$ & 133 & $\mathrm{~b}$ & 88 & $\mathrm{~b}$ & 133 & d & 5883 & $\mathrm{~b}$ & 8520 & $\mathrm{a}$ \\
\hline GI $036 \times$ TC2 & 75 & $\mathrm{c}$ & 258 & $\mathrm{a}$ & 185 & $\mathrm{~b}$ & 223 & g & 140 & a & 80 & $\mathrm{~b}$ & 124 & d & 5385 & b & 6444 & b \\
\hline GI $045 \times$ TC2 & 75 & $\mathrm{c}$ & 240 & $\mathrm{a}$ & 210 & $\mathrm{a}$ & 223 & $\mathrm{~g}$ & 143 & a & 105 & $\mathrm{a}$ & 126 & d & 6295 & b & 8338 & $\mathrm{a}$ \\
\hline GI $048 \times$ TC2 & 71 & $\mathrm{c}$ & 238 & $\mathrm{a}$ & 198 & $\mathrm{~b}$ & 200 & j & 148 & a & 93 & $\mathrm{~b}$ & 108 & $\mathrm{e}$ & 3812 & d & 5585 & $\mathrm{c}$ \\
\hline GI 049 x TC2 & 74 & $\mathrm{c}$ & 223 & $\mathrm{a}$ & 178 & $\mathrm{~b}$ & 219 & $\mathrm{~h}$ & 138 & $\mathrm{a}$ & 93 & $\mathrm{~b}$ & 119 & $\mathrm{~d}$ & 6734 & $\mathrm{a}$ & 7432 & b \\
\hline GI $088 \times$ TC2 & 74 & $\mathrm{c}$ & 223 & $\mathrm{a}$ & 183 & $\mathrm{~b}$ & 226 & $\mathrm{~g}$ & 133 & b & 88 & $\mathrm{~b}$ & 163 & $\mathrm{~b}$ & 5152 & $\mathrm{c}$ & 7304 & b \\
\hline GI $101 \times$ TC2 & 77 & $\mathrm{c}$ & 230 & $\mathrm{a}$ & 185 & $\mathrm{~b}$ & 236 & $\mathrm{f}$ & 148 & $\mathrm{a}$ & 90 & $\mathrm{~b}$ & 133 & d & 4516 & $\mathrm{c}$ & 5720 & b \\
\hline GI $104 \times$ TC2 & 73 & $\mathrm{c}$ & 248 & $\mathrm{a}$ & 190 & $\mathrm{~b}$ & 209 & 1 & 148 & $\mathrm{a}$ & 90 & $\mathrm{~b}$ & 110 & $\mathrm{e}$ & 5790 & $\mathrm{~b}$ & 5550 & c \\
\hline GI $105 \times$ TC2 & 77 & $\mathrm{c}$ & 228 & $\mathrm{a}$ & 203 & $\mathrm{a}$ & 223 & $\mathrm{~g}$ & 138 & $\mathrm{a}$ & 103 & $\mathrm{~b}$ & 131 & d & 6017 & b & 6727 & b \\
\hline GI $133 \times$ TC2 & 70 & $\mathrm{c}$ & 243 & $\mathrm{a}$ & 185 & $\mathrm{~b}$ & 195 & $\mathrm{j}$ & 138 & $\mathrm{a}$ & 95 & $\mathrm{~b}$ & 110 & e & 5477 & $\mathrm{~b}$ & 10188 & $\mathrm{a}$ \\
\hline GI 135 x TC2 & 77 & $\mathrm{c}$ & 265 & $\mathrm{a}$ & 188 & $\mathrm{~b}$ & 243 & $\mathrm{e}$ & 150 & $\mathrm{a}$ & 93 & $\mathrm{~b}$ & 140 & $\mathrm{c}$ & 5987 & $\mathrm{~b}$ & 7945 & \\
\hline
\end{tabular}


continuation

\begin{tabular}{|c|c|c|c|c|c|c|c|c|c|c|c|c|c|c|c|c|c|c|}
\hline GI $140 \times$ TC2 & 75 & c & 238 & $\mathrm{a}$ & 203 & $\mathrm{a}$ & 205 & $\mathrm{i}$ & 135 & $\mathrm{a}$ & 108 & $\mathrm{a}$ & 105 & e & 7463 & $\mathrm{a}$ & 4242 & $\mathrm{c}$ \\
\hline GI $148 \times$ TC2 & 73 & $\mathrm{c}$ & 253 & $\mathrm{a}$ & 183 & b & 238 & $\mathrm{e}$ & 150 & $\mathrm{a}$ & 88 & $\mathrm{~b}$ & 138 & $\mathrm{c}$ & 7129 & $\mathrm{a}$ & 8770 & $\mathrm{a}$ \\
\hline GI $149 \times$ TC2 & 74 & $\mathrm{c}$ & 248 & $\mathrm{a}$ & 195 & b & 219 & $\mathrm{~h}$ & 148 & $\mathrm{a}$ & 90 & $\mathrm{~b}$ & 111 & 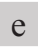 & 6399 & b & 8246 & 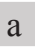 \\
\hline GI $150 \times$ TC2 & 73 & $\mathrm{c}$ & 238 & $\mathrm{a}$ & 195 & b & 225 & g & 145 & $\mathrm{a}$ & 93 & $\mathrm{~b}$ & 125 & d & 5174 & $\mathrm{c}$ & 8558 & $\mathrm{a}$ \\
\hline GI $151 \times$ TC2 & 75 & $\mathrm{c}$ & 245 & $\mathrm{a}$ & 188 & b & 230 & $\mathrm{f}$ & 140 & $\mathrm{a}$ & 98 & $\mathrm{~b}$ & 128 & d & 6328 & b & 8694 & $\mathrm{a}$ \\
\hline GI $152 \times$ TC2 & 72 & $\mathrm{c}$ & 258 & $\mathrm{a}$ & 183 & b & 225 & $\mathrm{~g}$ & 158 & $\mathrm{a}$ & 85 & $\mathrm{~b}$ & 122 & d & 4452 & $\mathrm{c}$ & 7549 & b \\
\hline GI $153 \times$ TC2 & 72 & $\mathrm{c}$ & 263 & $\mathrm{a}$ & 193 & b & 238 & e & 160 & a & 93 & $\mathrm{~b}$ & 145 & $\mathrm{c}$ & 6602 & $\mathrm{a}$ & 7624 & b \\
\hline GI 154 x TC2 & 75 & $\mathrm{c}$ & 240 & $\mathrm{a}$ & 173 & b & 237 & $\mathrm{f}$ & 145 & $\mathrm{a}$ & 75 & $\mathrm{~b}$ & 140 & $\mathrm{c}$ & 6300 & b & 7538 & 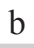 \\
\hline GI $155 \times$ TC2 & 76 & $\mathrm{c}$ & 233 & $\mathrm{a}$ & 193 & b & 231 & $\mathrm{f}$ & 135 & a & 108 & a & 128 & d & 4722 & $\mathrm{c}$ & 6003 & b \\
\hline GI $156 \times$ TC2 & 72 & $\mathrm{c}$ & 233 & $\mathrm{a}$ & 183 & b & 227 & g & 135 & a & 85 & $\mathrm{~b}$ & 118 & d & 5992 & $b$ & 8418 & $\mathrm{a}$ \\
\hline GI $157 \times$ TC2 & 78 & $\mathrm{c}$ & 258 & $\mathrm{a}$ & 190 & b & 230 & $\mathrm{f}$ & 148 & a & 95 & $\mathrm{~b}$ & 128 & d & 4744 & c & 7202 & b \\
\hline GI $158 \times$ TC2 & 75 & $\mathrm{c}$ & 240 & $\mathrm{a}$ & 180 & b & 225 & g & 148 & $\mathrm{a}$ & 88 & $\mathrm{~b}$ & 124 & d & 6680 & a & 5819 & $b$ \\
\hline GI $173 \times$ TC2 & 74 & $\mathrm{c}$ & 235 & $\mathrm{a}$ & 180 & b & 232 & $\mathrm{f}$ & 140 & $\mathrm{a}$ & 85 & $\mathrm{~b}$ & 134 & $\mathrm{c}$ & 6438 & a & 10104 & $\mathrm{a}$ \\
\hline \multirow{3}{*}{ Check Varieties } & \multicolumn{2}{|c|}{$\mathrm{FF}$} & \multicolumn{6}{|c|}{$\mathrm{PH}$} & \multicolumn{6}{|c|}{$\mathrm{EH}$} & \multicolumn{4}{|c|}{ GY } \\
\hline & \multicolumn{2}{|c|}{ (days) } & \multicolumn{6}{|c|}{$(\mathrm{cm})$} & \multicolumn{6}{|c|}{$(\mathrm{cm})$} & \multicolumn{4}{|c|}{$\left(\mathrm{kg} \mathrm{ha}^{-1}\right)$} \\
\hline & \multicolumn{2}{|c|}{ Means } & \multicolumn{2}{|c|}{ LD } & \multicolumn{2}{|c|}{$\mathrm{PB}$} & \multicolumn{2}{|c|}{$\mathrm{PG}$} & \multicolumn{2}{|l|}{ LD } & \multicolumn{2}{|c|}{ PB } & \multicolumn{2}{|c|}{ PG } & \multicolumn{2}{|l|}{$\mathrm{PB}$} & \multicolumn{2}{|l|}{ PG } \\
\hline IPV 2122 & 75 & $\mathrm{c}$ & 206 & $\mathrm{a}$ & 180 & $b$ & 206 & $\mathrm{i}$ & 113 & $\mathrm{c}$ & 75 & $\mathrm{~b}$ & 113 & $\mathrm{e}$ & 8989 & $\mathrm{a}$ & 7901 & $\mathrm{a}$ \\
\hline IPR 114 & 74 & $\mathrm{c}$ & 234 & $\mathrm{a}$ & 168 & b & 234 & $\mathrm{f}$ & 125 & b & 90 & $\mathrm{~b}$ & 125 & d & 6713 & a & 7640 & b \\
\hline IPR 115 & 74 & $\mathrm{c}$ & 226 & $\mathrm{a}$ & 170 & $\mathrm{~b}$ & 226 & $\mathrm{~g}$ & 130 & $\mathrm{~b}$ & 88 & $\mathrm{~b}$ & 130 & $\mathrm{~d}$ & 6946 & $\mathrm{a}$ & 8967 & $\mathrm{a}$ \\
\hline General Means & 75 & & 236 & & 193 & & 228 & & 139 & & 98 & & 129 & & 6052 & & 7185 & \\
\hline CV (\%) & & & 9,2 & & 7,6 & & 1,5 & & 7,9 & & 13,4 & & 7,7 & & 10,5 & & 19,8 & \\
\hline
\end{tabular}

Means followed by the same letter do not differ by the Scott-Knott test $(\mathrm{p}<0.05)$.

In the joint partial diallel analysis (Table 4), we observed that there were significant differences between the QM's for the sources of variation (SV), treatments $(\mathrm{T})$ and the parents $2(\mathrm{P} 2)$ for all of the variables analyzed, indicating that there is genetic variability among the evaluated populations. For Parental 1 (P1), a group composed of the test populations, there was no significance $(\mathrm{p}>0.05)$ of any traits. The Parents 2 (P2), a group composed of 28 maize landraces, exhibited significant differences for all traits, inferring that the maize landrace populations differ in their average allelic frequencies, contributing differently at intersections where they are involved. The groups (Gru) presented significant differences for the $\mathrm{PH}$ and GY, indicating that there was different behavior among them. In relation to the split of the QM's of the effects of heterosis in medium heterosis $(\overline{\mathrm{h}})$, a significant difference occurred for the $\mathrm{PH}$ and $\mathrm{EH}$, evidencing that the populations in crossbreeding obtained averages higher than the populations per se. Parental heterosis 2 (HP2) presented significant differences only for female flowering (FF). The absence of significance for heterosis of parents presented by $\mathrm{PH}, \mathrm{EH}$ and GY indicated that groups of Parental 1 (P1), testers, Parents (P2) and maize landraces populations when crossbreed do not present heterotic potential $\left(\mathrm{s}_{\mathrm{ij}}\right)$. Araújo and Miranda Filho (2001) also did not find significant differences for heterosis of varieties and total heterosis for the same traits analyzed in this work. 
Table 4. Mean squares (MS) in the diallel analysis for female flowering (FF), plant height (PH), ear height (EH) and grain yield (GY) applicable to partial diallels in several environments (Oliveira et al., 1987) Pato Branco, Ponta Grossa and Londrina-PR. Season 2008/2009.

\begin{tabular}{|c|c|c|c|c|c|c|c|c|c|c|}
\hline \multirow{3}{*}{ Source of Variation } & \multirow{3}{*}{ df } & \multicolumn{6}{|c|}{ Mean squares (MS) } & \multirow{3}{*}{$\mathrm{df}^{(\mathrm{a})}$} & \multirow{2}{*}{\multicolumn{2}{|c|}{$\begin{array}{l}\text { MS } \\
\text { GY }\end{array}$}} \\
\hline & & FF & & $\mathrm{PH}$ & & $\mathrm{EH}$ & & & & \\
\hline & & \multicolumn{2}{|l|}{ (Days) } & \multicolumn{4}{|c|}{$(\mathrm{cm})$} & & \multicolumn{2}{|l|}{$\left(\mathrm{kg} \mathrm{ha}^{-1}\right)$} \\
\hline Environments (E) & 2 & 1819.27 & $* *$ & 25784.76 & $* *$ & 38147.49 & $* *$ & 1 & 33982.03 & $* *$ \\
\hline Treatments (T) & 85 & 38.17 & $* *$ & 527.43 & $* *$ & 250.29 & ** & 85 & 3713.50 & $* *$ \\
\hline Parental $1(\mathrm{P} 1)$ & 1 & 0.02 & ns & 225.33 & ns & 261.33 & ns & 1 & 1007.31 & ns \\
\hline Parental 2 (P2) & 27 & 36.24 & $* *$ & 607.54 & $* *$ & 431.66 & $* *$ & 27 & 3385.59 & $* *$ \\
\hline Groups (Gru) & 1 & 8.37 & ns & 11992.03 & $* *$ & 257.19 & ns & 1 & 164125.59 & $* *$ \\
\hline Heterosis $(\mathrm{H})$ & 56 & 40.31 & $* *$ & 289.47 & ns & 162.52 & ns & 56 & 1055.42 & ns \\
\hline Means heterosis $(\mathrm{MH})$ & 1 & 14.32 & ns & 6975.03 & $* *$ & 2246.28 & $* *$ & 1 & 2878.92 & ns \\
\hline Parental heterosis 1 (PH1) & 1 & 3.24 & ns & 48.76 & ns & 0.76 & ns & 1 & 1883.72 & ns \\
\hline Parental heterosis 2 (PH2) & 27 & 34.92 & $* *$ & 205.50 & ns & 140.56 & ns & 27 & 1010.14 & ns \\
\hline Specific heterosis (SH) & 27 & 48.04 & ns & 134.74 & ns & 113.29 & ns & 27 & 1002.49 & ns \\
\hline $\mathrm{E} \times \mathrm{T}$ & 170 & 39.72 & $* *$ & 364.34 & ns & 277.68 & $* *$ & 85 & 1360.19 & ns \\
\hline Ex P1 & 2 & 14.85 & ns & 1513.13 & $* *$ & 796.91 & $* *$ & 1 & 2519.73 & ns \\
\hline E x P2 & 54 & 26.20 & ** & 133.56 & ns & 151.80 & ns & 27 & 672.54 & ns \\
\hline E x Gru & 2 & 274.18 & $* *$ & 14422.70 & $* *$ & 7972.58 & $* *$ & 1 & 1702.16 & ns \\
\hline Ex H & 112 & 42.49 & ** & 204.06 & ns & 191.69 & ** & 56 & 1664.93 & $*$ \\
\hline Ex MH & 2 & 105.02 & $* *$ & 2306.66 & $* *$ & 1054.26 & $* *$ & 1 & 342.00 & ns \\
\hline E x PH1 & 2 & 7.40 & ns & 98.38 & ns & 71.77 & ns & 1 & 1806.40 & ns \\
\hline E x PH2 & 54 & 36.63 & $* *$ & 178.47 & ns & 182.27 & ns & 27 & 1710.75 & $*$ \\
\hline Ex SH & 54 & 47.34 & ns & 155.69 & ns & 173.60 & ns & 27 & 1662.86 & ns \\
\hline Error & 255 & 15.85 & & 212.43 & & 133.00 & & 170 & 1088.42 & \\
\hline Means & & 73 & & 199 & & 109 & & & 5716 & \\
\hline
\end{tabular}

In relation to the estimates of varietal effects $\left(\hat{\mathrm{v}}_{\mathrm{j}}\right)$, the GI 018 population was highlighted because it presented the lowest $\hat{\mathrm{v}}_{\mathrm{i}}$ estimate for $\mathrm{FF}$, contributing to the reduction of the cycle by up to 6 days (Table 5). For PH, the populations GI 150 and GI 133 stood out with negative $\hat{\mathrm{v}}_{\mathrm{i}}$ estimates of 20 $\mathrm{cm}$, indicating that these populations, on average, contributed to the reduction in plant height. The lowest $\hat{\mathrm{v}}_{\mathrm{i}}$ estimate for $\mathrm{EH}$ was observed once again in the GI 150 population $(-23 \mathrm{~cm})$. The highest $\hat{\mathrm{v}}_{\mathrm{i}}$ estimates for GY were observed for the GI 148, GI 049 and GI 155 populations. The GI 133 stood out for the purpose of varietal estimates of most promising varieties for all traits, negatives estimates for FF (-4 days), PH $(-20 \mathrm{~cm}), \mathrm{EH}(-21 \mathrm{~cm})$ and positives estimates for GY $\left(1,015 \mathrm{~kg} \mathrm{ha}^{-1}\right)$, allowing its indication for recurrent selection programs or obtaining compounds. The effect of varieties is the performance of the " $i$ " population, performance $p e r$ $s e$, in relation to the average of the other populations, which is related to the additive components of the averages (GARDNER; EBERHART, 1966). According to HALLAUER et al. (1988), for the PH and $\mathrm{FF}$, negative and smaller values for parameter estimates are highly desirable, since they contribute to the additive model, reducing the mean $\mathrm{PH}$ and FF of the populations. Thus, it is valuable to select genotypes with negative estimates that contribute to the reduction of FF, PH and EH (SOLALINDE et al., 2014). For $\mathrm{PH}$ and $\mathrm{EH}$, there is a predominance of additive effects and only partial dominance (GORGULHO; MIRANDA FILHO 2001). 
The parental heterosis parameter $\left(\hat{\mathrm{h}}_{\mathrm{i}}\right)$ to $\mathrm{FF}$ oscillated between -13 days (GI 036) to 4 days (GI 018), wherein the GI 036 population stood out by having the lower estimate. For $\mathrm{PH}$ and $\mathrm{EH}$, the GI $088(-22 \mathrm{~cm})$ and GI $036(-14 \mathrm{~cm})$ populations were outstanding, with the most negative values. The highest estimates of the effects of heterosis on GY were presented by the GI $018\left(1121 \mathrm{~kg} \mathrm{ha}^{-1}\right)$ and GI 105 (775.32 $\mathrm{kg} \mathrm{ha}^{-1}$ ) populations. Two populations, GI 088 and GI 173, stood out as having promising estimates for all variables, low and negative for FF, $\mathrm{PH}$ and $\mathrm{EH}$, and high and positive for GY (Table 5), inferring that these populations in crossing will result in more heterotic hybrid combinations (CRUZ et al., 2004). According to Bernini and Paterniani (2012), the parental heterosis parameter is the difference between the mean of the heterosis of the hybrids and the mean heterosis.

For general combining ability $\left(\hat{\mathrm{g}}_{\mathrm{i}}\right)$, the GI 088 , GI 133 and GI 156 populations revealed promising negative estimates, contributing to a reduction in FF, PH and EH (Table 5). These same populations exhibited promising and significant $\hat{\mathrm{g}}_{\mathrm{i}}$ estimates toward increasing GY as GI $133\left(963 \mathrm{~kg} \mathrm{ha}^{-1}\right)$, GI 156 (439 kg ha-1) and GI 088 (373 kg ha-1). The general combining ability is a genetic parameter obtained due to the genetic differences in the parents and the average effects of an allelic substitution being associated with additive effects (HALAUER; MIRANDA FILHO, 1988). Kostetzer et al. (2009), evaluating local varieties of Paraná and synthetic maize varieties, identified promising $\hat{\mathrm{g}}_{\mathrm{i}}$ estimates in the following varieties: MC45 $\left(0,33 \mathrm{~kg} \mathrm{ha}^{-1}\right)$ in
São João do Triunfo and IAPAR $50\left(0,59 \mathrm{~kg} \mathrm{ha}^{-1}\right)$ in Londrina. Ferreira et al. (2009), evaluated 31 maize landrace populations in two locations in the state of Paraná, obtained from Londrina, and their estimates ranged from $-0,670 \mathrm{~kg} \mathrm{ha}^{-1}$ (MC 13) to $980 \mathrm{~kg}$ ha $^{-1}$ (MC 31). Senhorinho et al. (2015) evaluated common maize cultivars and found that $\hat{\mathrm{g}}_{\mathrm{i}}$ affects the promise for increasing GY in 30B39, 30K64 and 30B30, indicating the difference in the vigor of the maize landrace populations in comparison with modern maize cultivars. Oliboni et al. (2013) evaluated maize commercial cultivars and selected the hybrids P30F44 and 2B688 for the potential confirmed by the high $\hat{\mathrm{g}}_{\mathrm{i}}$ estimates obtained for grain yield and plant height.

In pre-breeding, it is difficult to gather genotypes that present promising genetic parameters for all evaluated traits, thus requiring selection. This is even more true when working with maize landrace populations that present very different traits. However, the $\hat{g}_{\mathrm{i}}$ estimates are associated with the frequency of favorable alleles for traits of agronomic interest (VENCOVSKY; BARRIGA, 1992), emphasizing that the positive estimates reflect the genotype for the contribution to an increase or a negative reduction in the trait. Thus, the maize landrace population GI 133 brought together the best effects of varieties $\left(\hat{\mathrm{v}}_{\mathrm{i}}\right)$ and general ability combining $\left(\hat{\mathrm{g}}_{\mathrm{i}}\right)$, allowing for use in recurrent selection programs or in obtaining hybrids and composites. The GI 088 population has collected the best estimates that can also be used in recurrent selection programs and for incorporation into hybrid combinations. 
Table 5. Estimates of the effects of population $\left(v_{i}\right.$ and $\left.v_{j}\right)$, parental heterosis $\left(h_{i}\right.$ and $\left.h_{j}\right)$ and general combining ability GCA $\left(g_{\mathrm{i}}\right.$ and $\mathrm{g}_{\mathrm{j}}$ ) on 28 maize landrace populations evaluated in Londrina, Pato Branco and Ponta Grossa-PR. Season 2008/09.

\begin{tabular}{|c|c|c|c|c|c|c|c|c|c|c|c|c|c|c|}
\hline \multirow{3}{*}{$\mathrm{V}(1)$} & $\mathrm{FF}$ & $\mathrm{PH}$ & EH & GY & \multirow{3}{*}{$\mathrm{V}(1)$} & $\mathrm{FF}$ & $\mathrm{AP}$ & $\mathrm{AE}$ & MG & \multirow{3}{*}{$\mathrm{V}(1)$} & $\mathrm{FF}$ & $\mathrm{PH}$ & $\mathrm{EH}$ & GY \\
\hline & \multicolumn{4}{|c|}{$\hat{\mathrm{v}}_{\mathrm{i}}$} & & \multicolumn{4}{|c|}{$\hat{\mathrm{h}}_{\mathrm{i}}$} & & \multicolumn{4}{|c|}{$\hat{g}_{i}$} \\
\hline & days & \multicolumn{2}{|c|}{$\mathrm{cm}$} & $\mathrm{kg} \mathrm{ha}^{-1}$ & & days & \multicolumn{2}{|c|}{$\mathrm{cm}$} & $\mathrm{kg} \mathrm{ha}^{-1}$ & & days & \multicolumn{2}{|c|}{$\mathrm{cm}$} & $\mathrm{kg} \mathrm{ha}^{-1}$ \\
\hline PC 0201 & 0,67 & $-4,83$ & -2 & $-464,5$ & PC 0201 & $-0,39$ & 1,52 & $-0,19$ & 366,81 & PC 0201 & $-0,06$ & $-0,89$ & $-1,19$ & 134,56 \\
\hline PC 0202 & $-0,67$ & 4,83 & 2 & 464,5 & PC 0202 & 0,39 & $-1,52$ & 0,19 & $-366,81$ & PC 0202 & 0,06 & 0,89 & 1,19 & $-134,56$ \\
\hline$V(2)$ & & \multicolumn{2}{|r|}{$\hat{\mathrm{v}}_{\mathrm{j}}$} & \multicolumn{2}{|r|}{$\mathrm{V}(2)$} & \multicolumn{3}{|c|}{$\hat{\mathrm{h}}_{\mathrm{j}}$} & \multicolumn{2}{|r|}{$\mathrm{V}(2)$} & \multicolumn{4}{|c|}{$\hat{\mathrm{g}}_{\mathrm{j}}$} \\
\hline GI 002 & 3 & -10 & -7 & -488 & GI 002 & 1 & -7 & 2 & -1278 & GI 002 & $2 * *$ & $-12 * *$ & -2 & $-1522 * *$ \\
\hline GI 006 & 3 & 19 & 14 & 656 & GI 006 & 0 & -6 & -5 & -409 & GI 006 & $1^{* *}$ & $4 * *$ & 1 & -81 \\
\hline GI 008 & 1 & 9 & 6 & 316 & GI 008 & 2 & 7 & 5 & -75 & GI 008 & $2 * *$ & $12 * *$ & $8 * *$ & 83 \\
\hline GI 018 & -6 & 3 & 4 & -414 & GI 018 & 4 & 5 & 4 & 1121 & GI 018 & $1^{*}$ & $6 * *$ & $5^{* *}$ & $914 * *$ \\
\hline GI 028 & 4 & -20 & -7 & 246 & GI 028 & -2 & 7 & 3 & 189 & GI 028 & -1 & $-3^{*}$ & -1 & $312 *$ \\
\hline GI 036 & -1 & -5 & -1 & -822 & GI 036 & -13 & 8 & -14 & -1155 & GI 036 & $-13 * *$ & $5 * *$ & $-15^{* *}$ & $-1566 * *$ \\
\hline GI 045 & -3 & -7 & -9 & 207 & GI 045 & 3 & 2 & 1 & 205 & GI 045 & $1 * *$ & -2 & $-3 * *$ & $309 *$ \\
\hline GI 048 & -2 & -13 & -19 & -2117 & GI 048 & -1 & -3 & 7 & -134 & GI 048 & $-2 * *$ & $-10 * *$ & $-2^{*}$ & $-1192 * *$ \\
\hline GI 049 & -2 & 0 & 3 & 1400 & GI 049 & 1 & -5 & -6 & -487 & GI 049 & 0 & $-5 * *$ & $-5 * *$ & 213 \\
\hline GI 088 & -1 & 1 & 1 & -726 & GI 088 & 0 & -22 & 1 & 737 & GI 088 & $-1 *$ & $-21 * *$ & $2^{*}$ & $373 * *$ \\
\hline GI 101 & 1 & -6 & -2 & -1269 & GI 101 & 1 & 0 & 5 & -446 & GI 101 & $1^{*}$ & $-2^{*}$ & $4 * *$ & $-1080 * *$ \\
\hline GI 104 & -1 & -13 & -10 & -606 & GI 104 & 1 & 10 & 8 & -377 & GI 104 & 1 & $4 * *$ & $3 * *$ & $-680 * *$ \\
\hline GI 105 & -1 & -11 & -9 & -2019 & GI 105 & 2 & 4 & 9 & 775 & GI 105 & $1 * *$ & -1 & $5 * *$ & $-234 *$ \\
\hline GI 133 & -4 & -20 & -21 & 1015 & GI 133 & 1 & -2 & -2 & 456 & GI 133 & $-1 *$ & $-12 * *$ & $-12 * *$ & $963 * *$ \\
\hline GI 135 & 0 & 19 & 27 & -936 & GI 135 & 0 & 8 & -4 & 365 & GI 135 & 1 & $17 * *$ & $9 * *$ & -103 \\
\hline GI 140 & 2 & 9 & 10 & 900 & GI 140 & 1 & -9 & -8 & -611 & GI 140 & $3 * *$ & $-4 * *$ & $-3 * *$ & -161 \\
\hline GI 148 & 4 & 4 & 3 & 2122 & GI 148 & -2 & 3 & -1 & 471 & GI 148 & 0 & $6 * *$ & 1 & $1531 * *$ \\
\hline GI 149 & 2 & 23 & 20 & 989 & GI 149 & -1 & -9 & -12 & 247 & GI 149 & 0 & $3^{*}$ & $-2 * *$ & $742 * *$ \\
\hline GI 150 & -3 & -21 & -23 & -789 & GI 150 & 1 & 6 & 8 & 524 & GI 150 & -1 & $-4 * *$ & $-4 * *$ & 129 \\
\hline GI 151 & 2 & 9 & 7 & 604 & GI 151 & -1 & 1 & 0 & -481 & GI 151 & 0 & $5 * *$ & $3 * *$ & -179 \\
\hline GI 152 & -1 & 8 & 8 & -61 & GI 152 & 0 & 3 & -3 & 149 & GI 152 & 0 & $7 * *$ & 0 & 119 \\
\hline GI 153 & -1 & 10 & 11 & 849 & GI 153 & -1 & 3 & 1 & 424 & GI 153 & $-2 * *$ & $8^{* *}$ & $7 * *$ & $848 * *$ \\
\hline GI 154 & 1 & -1 & -7 & -1402 & GI 154 & 0 & 7 & 8 & 668 & GI 154 & 1 & $7 * *$ & $4 * *$ & -33 \\
\hline GI 155 & 2 & -11 & -4 & 1218 & GI 155 & 0 & 4 & 2 & -960 & GI 155 & $2 * *$ & -2 & -1 & $-351 * *$ \\
\hline GI 156 & -3 & 2 & -5 & 679 & GI 156 & 1 & -6 & -5 & 99 & GI 156 & $-1^{*}$ & $-5 * *$ & $-8 * *$ & $439 * *$ \\
\hline GI 157 & 2 & 7 & 2 & 35 & GI 157 & 0 & 0 & 0 & -793 & GI 157 & $2 * *$ & $3 * *$ & 0 & $-776 * *$ \\
\hline GI 158 & -3 & 9 & 4 & -149 & GI 158 & 3 & -9 & -3 & 373 & GI 158 & $2 * *$ & $-4 * *$ & -1 & $298^{*}$ \\
\hline GI 173 & 4 & 6 & 5 & 563 & GI 173 & -1 & -5 & 2 & 404 & GI 173 & 1 & -2 & $5^{* *}$ & $686 * *$ \\
\hline Means & 73 & 199.1 & 109.52 & 5716.19 & & & & & & & & & & \\
\hline D & -1.01 & -0.92 & -6.18 & 1567.81 & & & & & & & & & & \\
\hline Heterosis & 0.85 & 18.78 & 10.66 & 467.43 & & & & & & & & & & \\
\hline
\end{tabular}

\section{Conclusions}

Topcross hybrids exhibited productive potential and differential performance in the environments in which they were evaluated.

Maize landrace GI 133 and GI 173 populations presented per se positive potential for indication in recurrent selection programs or for participation in obtaining composites.

The GI 088 and GI 173 populations stood out in terms of the effects of heterosis $\left(\hat{\mathrm{h}}_{\mathrm{i}}\right)$, indicating that they are promising populations for maize breeding programs. 


\section{Acknowledgements}

We would like to acknowledge CAPES for granting master's scholarship to the first author.

\section{References}

ARAÚJO, P. M.; MIRANDA FILHO, J. B. Analysis of diallel cross for the evaluation of maize populations across enviroments. Crop Breeding And Applied Biotecnology, Londrina, v. 1, n. 3, p. 255-262, 2001.

ARAÚJO, P. M.; NASS, L. L. Caracterização e avaliação de populações de milho crioulo. Scientia Agricola, Piracicaba, v. 59, n. 3, p. 589-593, 2002.

BERNINI, C. B.; PATERNIANI, M. E. A. G. Z. Estimativas de parâmetros de heterose em híbridos de populações $\mathrm{F}_{2}$ de milho. Pesquisa Agropecuária Tropical, Goiânia, v. 42, n. 1, p. 56-62, 2012.

CANCELLIER, L. L.; AFFÉRRI, F. S.; DOTTO, M. A.; CARVAlHO, E. V. de; DUTRA, D. P.; CORNÉLIO, G. L. Avaliação de top crosses de milho no sul do Tocantins. Revista Brasileira de Ciências Agrárias, Lavras, v. 6, n. 4, p. 557-564, 2011.

CARPENTIERI-PÍPOLO, V.; SOUZA, A.; SILVA, D. A.; BARRETO, T. P.; GARBUGLIO, D. D.; FERREIRA, J. M. Avaliação de cultivares de milho crioulo em sistema de baixo nível tecnológico. Acta Scientiarum. Agronomy, Maringá, v. 32, n. 2, p. 229-233, 2010.

CLOVIS, L. R.; SCAPIM, C. A.; PINTO, R. J. B.; BOLSON, E.; SENHORINHO, H. J. C. Avaliação de linhagens $\mathrm{S}_{3}$ de milho por meio de testadores adaptados à safrinha. Revista Caatinga, Mossoró, v. 28, n. 1, p. 109120, 2015.

CRUZ, C. D. Programa Genes: análise dialélica completo. Viçosa: Editora UFV, 2006. 175 p.

CRUZ, C. D.; REGAZZI, A. J.; CARNEIRO, P. C. S. Modelos biométricos aplicados ao melhoramento genético. 3. ed. Viçosa: UFV, 2004. 480 p. v. 1.

FERREIRA, D. F. Aplicativo MAPGEN 3,0. Lavras: Ed. UFL, 1993.

FERREIRA, J. M.; MOREIRA, R. M. P.; HIDALGO, J. A. F. Capacidade combinatória e heterose em populações de milho crioulo. Ciência Rural, Santa Maria, v. 39, n. 2, p. 332-339, 2009.

FRITSCHE-NETO, F.; VIEIRA, R. A.; SCAPIM, C. A.; MIRANDA, G. V.; REZENDE, L. M. Updating the ranking of the coefficients of variation from maize experiments. Acta Scientiarum Agronomy, Maringá, v. 34, n. 1, p. 99-101, 2012.
GARBUGliO, D. D.; ARAÚJO, P. M. Avaliação de híbridos intervarietais de milho por meio de cruzamento dialélico parcial considerado quatro ambientes. Semina: Ciências Agrárias, Londrina, v. 27, n. 3, p. 375-392, 2006.

GARDNER, C. O.; EBERHART, S. A. Analysis and interpretation of the variety cross diallel and related populations. Biometrics, Washington, v. 22, n. 3, p. 439452, 1966.

GORGULHO, E. P.; MIRANDA FILHO, J. B. Estudo da capacidade combinatória de variedades de milho no esquema de cruzamento dialélico parcial. Bragantia, Campinas, v. 60, n. 1, p. 1-8, 2001.

GRIFFING, B. Concept of general and specific combining ability in relation to diallel crossing system. Australian Journal of Biological Sciences, Melbourne, v. 9, n. 4, p. 463-493, 1956.

HALlAUER, A. R.; MIRANDA FILHO, J. B. Quantitative genetics in maize breeding. 2. ed. Ames: The Iowa State University Press, Iowa: 1988. 468 p.

HALLAUER, A. R.; RUSSEL, W. A.; LAMKEY, K. R. Corn breeding. In: SPRAGUE, G. F.; DUDLEY, J. W. Corn and corn improvement. 3. ed. Madison: Agron Monogr 18, ASA, CSSA and SSSA, 1988. p. 463-564.

KOSTETZER, V.; MOREIRA, R. M. P.; FERREIRA, J. M. Cruzamento dialélico parcial entre variedades locais do Paraná e variedades sintéticas de milho. Pesquisa Agropecuária Brasileira, Brasília, v. 44, n. 9, p. 11521159, 2009.

MIRANDA FILHO, J. B.; GERALDI, I. O. Anadapted model for the analysis of partial diallel crosses. Revista Brasileira de Genética, Ribeirão Preto, v. 7, n. 4, p. 677688, 1984.

MIRANDA, G. V. COIMBRA, R. R.; GODOY, C. L.; SOUZA, L.V.; GUIMARÃES, L. J. M.; MELO, A. V. de. Potencial de melhoramento e divergência genética de cultivares de milho-pipoca. Pesquisa Agropecuária Brasileira, Brasília, v. 38, n. 6, p. 681-688, 2003.

OLIBONI, R.; FARIA, M. V.; NEUMANN, M.; RESENDE, J. T. V.; BATTISTELLI, G. M.; TEGONI, R. G.; OLIBONI, D. F. Diallelic analysis in assessing the potential of maize hybrids to generate base-populations for obtaining lines. Semina: Ciências Agrárias, Londrina, v. 34, n. 1, p. 7-18, 2013.

OLIVEIRA, A. C.; MORAIS, A. R.; SOUZA JUNIOR, C. L.; GAMA, E. E. G. Análise de cruzamentos dialélicos parciais repetidos em vários ambientes. Revista Brasileira de Genética, Ribeirão Preto, v. 10, n. 3, p. 517-533, 1987. 
PIMENTEL-GOMES, F. Curso de estatística experimental. 14. ed. Piracicaba: Livraria Nobel. 2000. $477 \mathrm{p}$.

SCOTT, A. J.; KNOTT, M. A cluster analysis method for grouping means in the analysis of variance. Biometrics, Raleigh, v. 30, n. 3, p. 507-512. 1974.

SEIFERT, A. L.; CARPENTIERI-PÍPOLO, V.; FERREIRA, J. M.; GERAGE, A. C. Análise combinatória de populações de milho pipoca em topcrosses. Pesquisa Agropecuária Brasileira, Brasília, v. 41, n. 5, p. 771-778, 2006.
SENHORINHO, H. J. C.; PINTO, R. J. B.; SCAPIM, C. A.; MILANI, K. F.; NIHEI, T. H. Combining abilities and inbreeding depression in commercial maize hybrids. Semina: Ciências Agrárias, Londrina, v. 36, n. 6, p. 4133-4150, 2015.

SOLALINDE, J. M. Q.; SCAPIM, C. A.; VIEIRA, R. A.; AMARAL JUNIOR, A. T.; VIVAS, M.; PINTO, R. J. B.; MORA, F.; VIANA, A. P. Performance of popcorn maize populations in South American Avatí Pichingá using diallel analysis. Australin Journal of Crop Science, Melbourne v. 8, n. 12, p. 1632-1638, 2014.

VENCOVSKY, R.; BARRIGA, P. Genética biométrica no fitomelhoramento. Ribeirão Preto: Sociedade Brasileira de Genética, 1992. 486 p. 